\title{
Watching To Learn: Implicit Learning of Motor Behavior Through Observation in Middle-School Students
}

\author{
Nayeric Cairo ${ }^{1}$, Janette Sierra ${ }^{1 \#}$ and Maryam Vaziri Pashkam ${ }^{1 \#}$ \\ ${ }^{1}$ Pinecrest Preparatory High School, Miami, FL, USA \\ \#Advisor
}

\section{ABSTRACT}

There is a disagreement in the neuropsychological literature regarding the nature of observational learning, strictly without a motor component. While some studies claim explicit influences are needed in order for learning to occur, others argue that they are not. The current student aims at understanding this gap, in particular emphasizing the observation aspect, since there is a limited number of research on this component. An experiment was conducted to measure both the short- and long-term of this effect using a neutral parameter procedure among two groups and a control. It was found that there was a significant effect of implicit learning. These findings are analyzed in a behavioral aspect and taken into consideration for limitations, implications and future research.

\section{Introduction}

For decades, the topic of learning has interested psychologists. More recently, research in this field has undergone critical development regarding a specific type of learning: observational. Although there are slightly varying definitions of the term across the literature, Maslovat, Hodges and Krigolson, a group of recognized Kinesiology professors at the Universities of Ottawa, British Columbia, and Victoria, correspondingly (2010), established a basic foundation, describing observational learning as "the process by which an observer adapts his or her movements as a result of watching a model" (p. 7).

Bandura (1986) explored modeling and cognition, and the role they play in understanding behavior, known as the social cognitive perspective. Moreover, Bandura (1971) proposed his cognitive mediation model, which established that solely observing leads to an internal representation of action and then elicits an action response. His behavior-focused work laid a foundation for future research and would be later supported by neuroimaging studies.

Nevertheless, there are still some relevant gaps in the literature regarding the nature of observational learning. A majority of studies corroborate the outlook that explicit processes, such as overt action, mediate observational learning (Rüssler \& Rösler, 2000). However, evidence in support of implicit observational learning, that is learning that takes place internally without any motor involvement, is scant.

Vinter and Perruchet (2002), had attempted to explore this phenomenon on adults and children; however, a motor factor was accounted for, making the task not solely implicit. Thus, the current study addresses this gap and aims at understanding whether implicit learning of a behavior through observation can take place when explicit influences are reduced. To do this, the study focuses on the population of middle-school students, in which this effect has not previously been measured. Understanding the effect of implicit learning among this population is critical for efficient applications in educational scenarios. 


\section{Literature Review}

In order to situate the gap found in the implicit learning literature that this study addresses, it is important to understand the related body of research that has led there. For these purposes, the review of the literature has been divided into two sections: neuropsychological and behavioral. These two fields of work, in combination, provide insight into what is known in the overall subject and show what areas have already been explored and those that are still in need of development. However, due to the nature of the present study, the behavioral aspect was the focus.

\subsection{Neuropsychological Work}

Bandura's (1986) work showed that not only is the use of demonstrations, or models, one of the most common and effective approaches to learning, but as demonstrated in his cognitive mediation model, through observing, mental representations are mapped internally, thereby improving the learning process. Further neuroimaging work has provided evidence for these findings, as researchers in cognitive psychology and neuroscience have been studying the relationship between action perception and motor processes.

Revolutionary work in this field includes the discovery of mirror neurons, the cells of the nervous system and their different locations across the nervous system, which together form the mirror neuron system or MNS (Lodish, 2000). These were first discovered in monkeys by di Pellegrino, et al. (1992), a group of renown neurophysiologists, and then in humans (Rizzolatti, et al., 2001) where the MNS engages the motor areas of the brain during observation of action, thus enabling individuals to understand others' behavior to imitate observed movements. The "direct matching" hypothesis allows individuals to "understand actions when we map the visual representation of the observed action onto our motor representation of the same action', (Rizzolatti, et al., 2014, p.12).

\subsection{Behavioral Work}

In the the behavioral literature, there is some disagreement as to whether observational learning is mediated by different systems, meaning implicitly or explicitly. Most authors agree that implicit learning is defined as a phenomenon that deals with unconscious processes, which are nonetheless evident in subsequent behavioral responses; whereas explicit learning is that which relies on the deliberate attempt and awareness of the acquisition that is taking place (Vinter \& Perruchet, 2002).

Though some studies, including Reber (1993) have found that performance in implicit learning tasks is not based on the use of conscious knowledge of the particular training circumstances, others assert that this explicit knowledge can influence performance. Reber employed an experimental design that consisted of training participants through a serial reaction time (SRT) task. In the classic SRT, participants respond to stimuli set that appears to be in random order but is actually repeating sub-sequences. These stimuli are usually presented on a computer screen, and the participant must press a key that corresponds to the location shown as fast as possible (Nissen \& Bullemer, 1987). Kelly \& Burton's (2001) version of this experiment used solely observation. They found that participants could successfully generate the presented sequence, thus demonstrating their conscious awareness of the learning that had taken place. The results suggested that while action-based research can be implicit, learning of an SRT task through observation is indeed explicit. Similarly, Howard et al. (1992) found that there were higher levels of awareness in participants learning by observation rather than action and concluded that the learning effect was due to explicit influences. On the neuropsychology side, Rüsseler and Rösler (2000) found that there was different neural activity between participants who learned an SRT task implicitly and explicitly. Overall, these studies support the perspective that observational learning is mediated by explicit processes.

On the other hand, there have been multiple studies that challenge this view (Bird, et al., 2005; Heyes \& Foster, 2002; Nissen \& Bullemer, 1987). Nissen and Bullemer (1987) introduced the SRT and found that this 
sequential knowledge was implicit by demonstrating that participants were unable to reproduce the sequence consciously without cues at the end of the training. This is in line with further research, including the Bird, et al. (2005) study, which countered Kelly and his colleagues' hypothesis, by demonstrating that overt action was not required for implicit learning to take place during observation. This connects to the aforementioned mental representations that are formed during the learning process, and the principle of early mediation, in which the participants implicitly involve neural systems during observational through mental representations, aided by the MNS (Heyes \& Foster, 2002).

Nevertheless, because of the disagreement in the literature, there is still a pertaining gap as to the true nature of learning through observation, strictly without a motor component. In fact, there have been very few studies in the literature that have explored this phenomenon.

\section{2a Behavioral Work: Implicit Learning Through Observation; Procedures}

An understanding of implicit learning through observation alone was partially attempted by Vinter $\&$ Perruchet, ${ }^{1}$ a group of developmental psychologists at the University of Bourgogne, Dijon, France, (2002) who similarly studied "whether implicit observational learning can occur when explicit influences on behavior are avoided" (p.2). In order to measure this effect, they had to come up with a technique which was free of overt influences: the neutral parameter procedure, which was then implemented in a simple drawing task of geometric figures they visually showed participants (Vinter \& Perruchet, 1999). This consists of a covariation, or manipulation, of a particular feature in the drawing task, that is the relationship between where a figure starts being drawn (top or bottom) and the direction in which the drawing proceeds (clockwise or counterclockwise). This is deliberately stated in the start-rotation principle (SRP), presented by van Sommers, a psychology professor at the University of Cambridge. (1984): in spontaneous drawing, when a figure starts from the top, it is predominantly drawn in a counterclockwise rotation, whereas when a figure starts from the bottom, it is predominantly drawn in a clockwise rotation. In their experiment, Vinter \& Perruchet (1999) inherently inverted this covariation, meaning when a figure was drawn starting at the top, the drawing counterintuitively progressed clockwise, and coincidently, when a figure was drawn starting at the bottom, the drawing progressed counterclockwise. Thus, by separating "the parameter on which unconsious effects are assessed" (inversion of the covariation) and "the parameter on which task achievement is assessed" (accuracy and successful detection of distractor), any learning that took place would have been implicit, and explicit influences would have been eliminated (Vinter \& Perruchet, 1999, p. 2).

However, their study was not purely observational. They asked participants to follow the drawings with their eyes, which is considered a motor component, as corroborated by Vogt (1995). Therefore, the exploration of this effect through observation alone is still needed. Furthermore, Vinter \& Perruchet had specifically looked at adults and children. As such, it is not yet known what the effect would be in middle school students, a population with peak cognitive development, as well as the potential educational benefits of better teaching strategies to help students retain material.

The present study adapted Vinter and Perruchet's (2002) procedure, but strictly without a motor component to answer: To what extent and with what longevity can implicit learning of a motor behavior take place through observation in middle school students?

Based on Vinter \& Perruchet (2001) and related studies, it is hypothesized that the participants will show evidence of implicit observational learning, even without a motor component. In all, the answers to the question this study will explore are of real-world relevance. By investigating this research question, the current study hopes to gain knowledge about not only learning in a particular controlled application, but also to get insight into the more broad educational and day-to-day applications of this process, since learning is everywhere. This understanding aims to inspire the use of more neurocognitive learning principles not only inside the classroom, but also in everyday life. 


\section{Methods}

For the purposes of this study, a fully experimental design with a quantitative collection and data analysis method was employed. This method was chosen because in order to measure the extent to which implicit learning (dependent variable) of a motor behavior through observation alone, a focus that had not been looked at before, can take place when explicit influences are reduced (independent variable), there had to be some manipulation. In this case, that is the covariation of the SRP principle. Thus, directly isolating the independent variable in a controlled environment while accounting for other variables that might affect the dependent variable allows for the most effective results of a cause-and-effect scenario. This is also supported by previous research and cognitive neuroscientist, Maryam VaziriPashkam, who was the expert adviser for this study.

\subsection{Participants}

In the current study, participants were selected through random sampling of $6^{\text {th }}-8^{\text {th }}$ grade students at a public charter middle school in Miami-Dade County, Florida. A total of 37 middle-school students (20 males, 17 females), aged 1113 years, (mean age 11.5 years) volunteered to take part in the observational training experiment. The ideal sample size was determined from Vinter \& Perruchet's (2002) original study and calculated using the Cochran's formula ${ }^{1}$. The participants were unaware of the study's purpose and were randomly assigned to one of three groups: $80 \%$ SRP conformity group $(\mathrm{N}=15), 20 \% \mathrm{SRP}$ conformity group $(\mathrm{N}=11)$ and a control group $(\mathrm{N}=11)$, which was not exposed to any form of the SRP, as established by van Sommers (1984). They were told that by participating in the experiment, they would be entered for a \$20 gift card raffle. There were three winners, spread evenly among the three groups, and

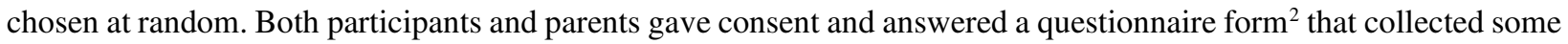
simple information, so that later it could be accounted in the data analysis. Here, the participants self-declared handedness (37 right-handed) and also confirmed they had no visual or psychomotor deficits as well as any form of educational advancement or retardation. Lastly, the study followed both ethical regulations and COVID-19 guidelines as established by the School Board.

\subsection{Material}

The experiment was run using an educational program called Seesaw that served as a platform for the participants to engage. To maximize hygiene and minimize physical contact, the participants used their own personal devices to operate the software. For that reason, the method of control of the device's pointer, includin mouse, trackpad, and touchscreen, was recorded in the questionnaire they had filled out prior, which could then be accounted for in data analysis. This program acted as both a digitizer and monitor screen, allowing participants to watch videos in the training phase and also record their responses directly over the figures presented in the test phase, using the aforementioned method of control over the device's pointer. The appearance of the figures in the screen was software controlled and was measured prior to the experiment. The figures included a square $(5 \mathrm{~cm} \times 5 \mathrm{~cm})$, rectangle $(5 \mathrm{~cm} \times 3 \mathrm{~cm})$, triangle $(5 \mathrm{~cm} \times 5 \mathrm{cmc} \times 5 \mathrm{~cm})$ and circle $(5 \mathrm{~cm}$ diameter $)$. They were all displayed in the center of the screen inside a $5 \mathrm{~cm} \times$ $5 \mathrm{~cm}$ square. Furthermore, the figures incorporated a starting point of a diameter of $0.1 \mathrm{~cm}$, which was located either at the top or bottom of the figure (Middle of the horizontals for the square and rectangle, 12 o'clock/6 o'clock positions for the circle, and top of the triangle or middle of its base), (See Figure 1).

\footnotetext{
${ }^{1}$ Cochran's formula is $\mathrm{n}_{0}=\underline{\mathrm{Z}^{2} \mathrm{pq}}$

${ }^{2}$ See Appendix. 




Figure 1. Shows an example of the figures, in this case, a circle, the participants were presented (Not displayed to scale).

\subsection{Procedure}

\section{3a Experiment 1: Short-Term Effect}

The experiment took place in a well-lit and comfortable room. It consisted of three phases: a training section, a test section, and a questionnaire section.

In the training section, participants in Group 1 (80\% SRP conformity group) and Group 2 (20\% SRP conformity group) were instructed to watch videos of each of the four shapes, thereby totaling four, which lasted about five minutes in total. Here, they were asked to pay attention and stay on the lookout for a pause, which lasted 3 seconds, since they would be asked afterwards to identify in the video of what shape did a pause appear. This is a key difference between the present study and the original Vinter \& Perruchet (2002) design. The adaption was made so that there would be no motor component at all, but it could still be used as a measurement of attention. The participants were given four options: square, rectangle, triangle and circle and were instructed to choose one. No time constraint was imposed, and they were given no feedback when done. The majority of the participants (77\%) perceived the pause in the video of the correct shape. However, this task solely served to ensure the participants were attentively watching the videos, since it made the observation of the drawing necessary, but without a motor component at all.

In Group 1 and Group 2 the participants watched a video for each of the four figures (square, rectangle, triangle, and circle), which showed 10 repetitions of the tracing, making a total of forty figures. The videos for Group 1 (80\% SRP conformity) contained 4 figures, one of each shape, that was traced following the SRP, for both starting points, thus totaling 8 tracings, which consisted of clockwise rotations when starting at the top, and counterclockwise rotations when starting at the bottom. The remaining $80 \%$ of the tracings for all four of the figures, applied the covariation of the principle. This meant 32 tracings, 4 of each shape, 16 of which started at the top, and 16 of which started at the bottom. For Group 2 (20\% SRP conformity), the videos displayed the exact opposite. Thus, there were in turn, the same number clockwise and counterclockwise rotations as well as top and bottom start. Furthermore, the order in which the videos were shown, and the combinations of starting points and directions was random across the participants.

In the test section, both groups were presented with the same screen they had seen in the videos, but this time instead of watching, they were instructed to trace directly over the figures, respecting the starting point at either the top or bottom. However, the direction of rotation of the drawing movement that was expected was not explained. There was a total of 24 figures consisting of six repetitions of each figure (square, rectangle, triangle, and circle), half starting at the top and half starting at the bottom. The software recorded participants responses as videos, coincidently including the direction of rotation and the starting point of the drawing. However, they were not able to manipulate anything on the screen. Participants were given no time constraint but finished on average after 5 minutes. 
Lastly, in the questionnaire phase, participants were asked to answer some questions regarding the videos they had watched and from what they recalled. They stated for all four figures, if the tracing of the figure started at either the top or the bottom, in which direction did it mostly progress in. They were asked to answer either clockwise or counterclockwise. Thus, there was a total of 16 questions. Moreover, the participants were invited to comment anything they had perceived during the experiment. This was used in the data analysis as a way to measure if the participants were aware of the covariation that had been employed in the experiment.

For the Control Group, since the participants were not exposed to any form of SRP, they did not watch any videos at all. This was used to test the accuracy of the principle itself in the same population. Instead, this group skipped the training phase, and went directly into the test phase, which was the same one from the other two groups. They also did not have to complete the following questionnaire.

\section{3b Experiment 1: Long-Term Effect}

Long-term effect was measured as well following a period of one day from the first part of the experiment. Here, the long-term effect was defined as the participants' ability to recall what they had learned implicitly and recreate such behaviors (Vinter \& Perruchet, 2002) The longevity of this effect had not been explored in the exact context that this study investigates before. Although there had been some studies that looked at the effect of an implicit memory task over time, they also contained a motor component (Blakemore, 1977). Therefore, the longstanding outcomes of implicit learning without a motor component, as this study aims to understand, still need to be explored because they are critical in applying the results in real-world scenarios.

The same procedures were employed as in the first part of the experiment. However, participants in Group 1 and Group 2 did not watch the videos this time because then they would not have been relying on memory. They also did not answer the questionnaire since, once again, they had not watched the videos. Thus, this would have conversely relied too much on memory and neither extremes were preferable. Furthermore, the control group did not skip any sections because they had not watched any videos in the first place, since they were not experimental. Finally, all the participants were debriefed about the purpose of the study and then the winners of the raffle were announced.

\section{Results}

To answer the research question the current study had proposed regarding implicit learning of a motor behavior solely through observation alone in both the short and long-term, two types of measurements were used, as established by the neutral parameter procedure. First, the actual drawing performances of the participants that had taken place after the training phase served as an assessment of the implicit learning that had occurred. Second, the questions the participants answered in the questionnaire phase provided an evaluation of their awareness of the manipulated covariation that had been employed. Together, both of these measurements were coded in terms of the SRP and analyzed, meaning a high percentage would signal a high conformity to the principle and vice-versa.

It was originally hypothesized that the participants would demonstrate signs of implicit learning, that is they would follow the direction in which the drawings they were shown in their corresponding videos mostly progressed in. Meaning, for instance, that Group 1 ( $80 \%$ SRP conformity group) should have a higher compliance to the SRP, as opposed to Group 2 (20\% SRP conformity group).

Therefore, the results were broken up into four categories: short term performance, short term awareness, long term performance and the relationships among these. As mentioned previously, long term awareness was not measured because this would have heavily relied on recall. 


\subsection{Implicit Performance}

In the first Test Phase, when participants in Group $1(\mathrm{~N}=15)$ were asked to execute the same task, they had watched in the videos immediately after they had seen them, they followed the SRP $63 \%$ of the time, as opposed to participants in Group 2 ( $\mathrm{N}=11$ ), who only followed the SRP $31 \%$ of the time (See Figure 2). To corroborate whether this difference was due to chance, a T-Test was conducted between the two groups, SRP conforming and SRP non-conforming. The 15 participants who watched videos that drew the majority of figures in congruity with the SRP $(M=.6278, S D=$ 0.2447) compared to the 11 of those that watched the videos that drew the majority of the figures in incongruity with the SRP $(M=.3106, S D=0.1701)$ demonstrated a significantly higher compliance with the SRP, $t(24)=3.6857, p<$ .001 , as had been hypothesized.

\section{1a Short Term Performance}

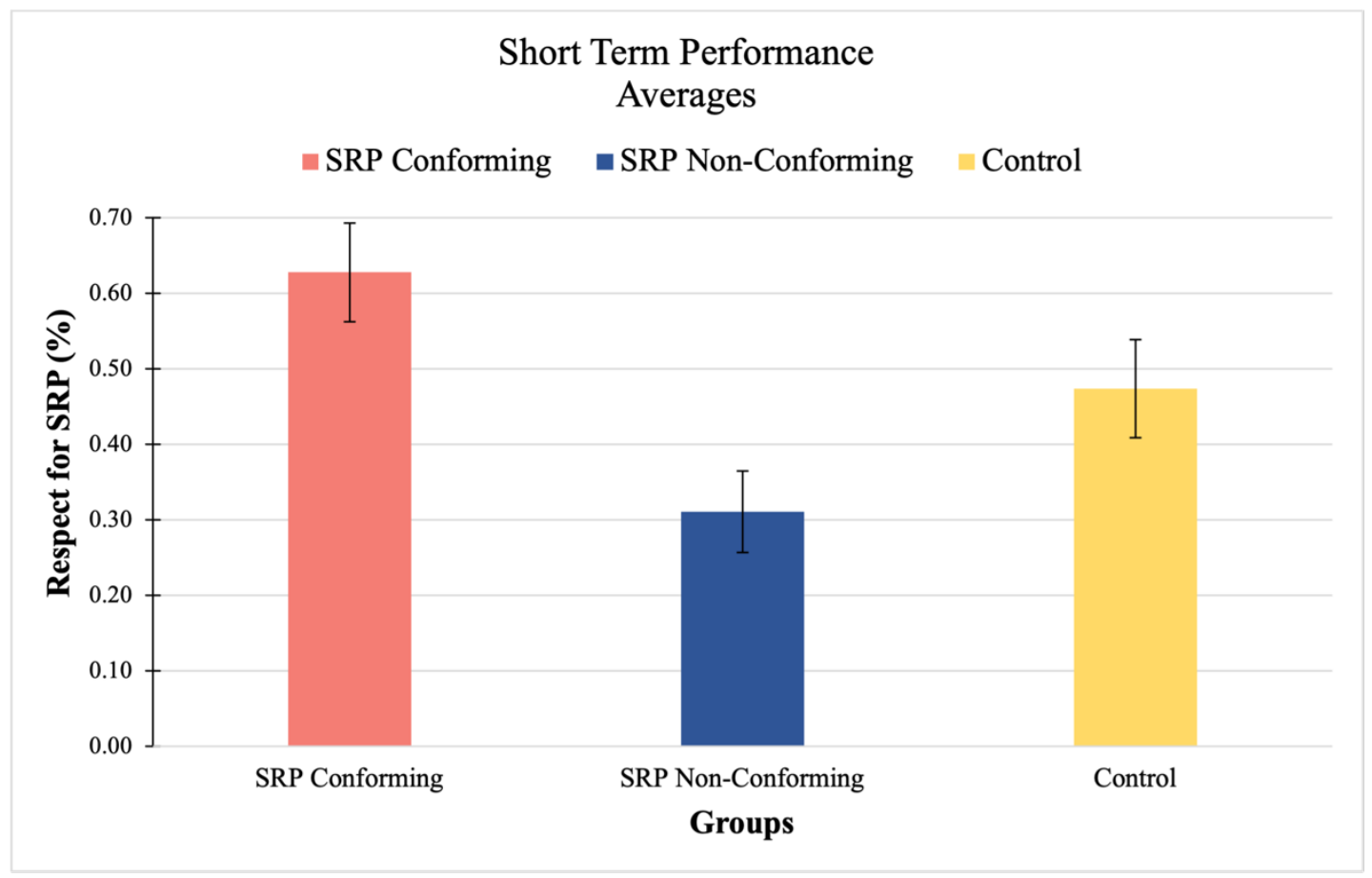

Figure 2. Chart of short-term performance averages for the SRP Conforming, SRP Non-Conforming, and the Control groups.

Further T-Tests were calculated between the experimental groups and Group 3, or the control (N=11). Here, a difference was reported between SRP non-conforming and control, $t(20)=2.0266, p=.0562$, however it was not statistically significant. Furthermore, the T-Test reported between SRP conforming and control, $t(24)=1.6967, p>$ .05 did not show a difference. This represents that although there was an effect of implicit learning through observation, the main effect of SRP was not as relevant. This may have occurred due to other influences that may have altered performance, such as the type of devices used by participants. This is further discussed in the limitations. 


\section{1b Long Term Performance}

To measure the longevity of the implicit learning effect in the long-term, another T-Test was conducted between Group 1 and Group 2 using their performances from the second Test Phase, which had taken place a day following the first one. Once again, it was demonstrated that the SRP conforming group imitated the principle as was shown in their videos more often (53\%) than did those in the SRP non-conforming group (35\%). More specifically, the 15 participants who watched videos that mostly followed the SRP $(M=.5361, S D=0.2308)$ compared to the 11 of those that watched the videos that mostly went against the SRP $(M=.3598, S D=0.2102)$ showed once more a significantly higher compliance with the SRP, $t(24)=2.0161, p=0.0574$. However, this difference was not nearly relevant as compared to the short-term performance, leading to the inference that the implicit learning effect fades with time.

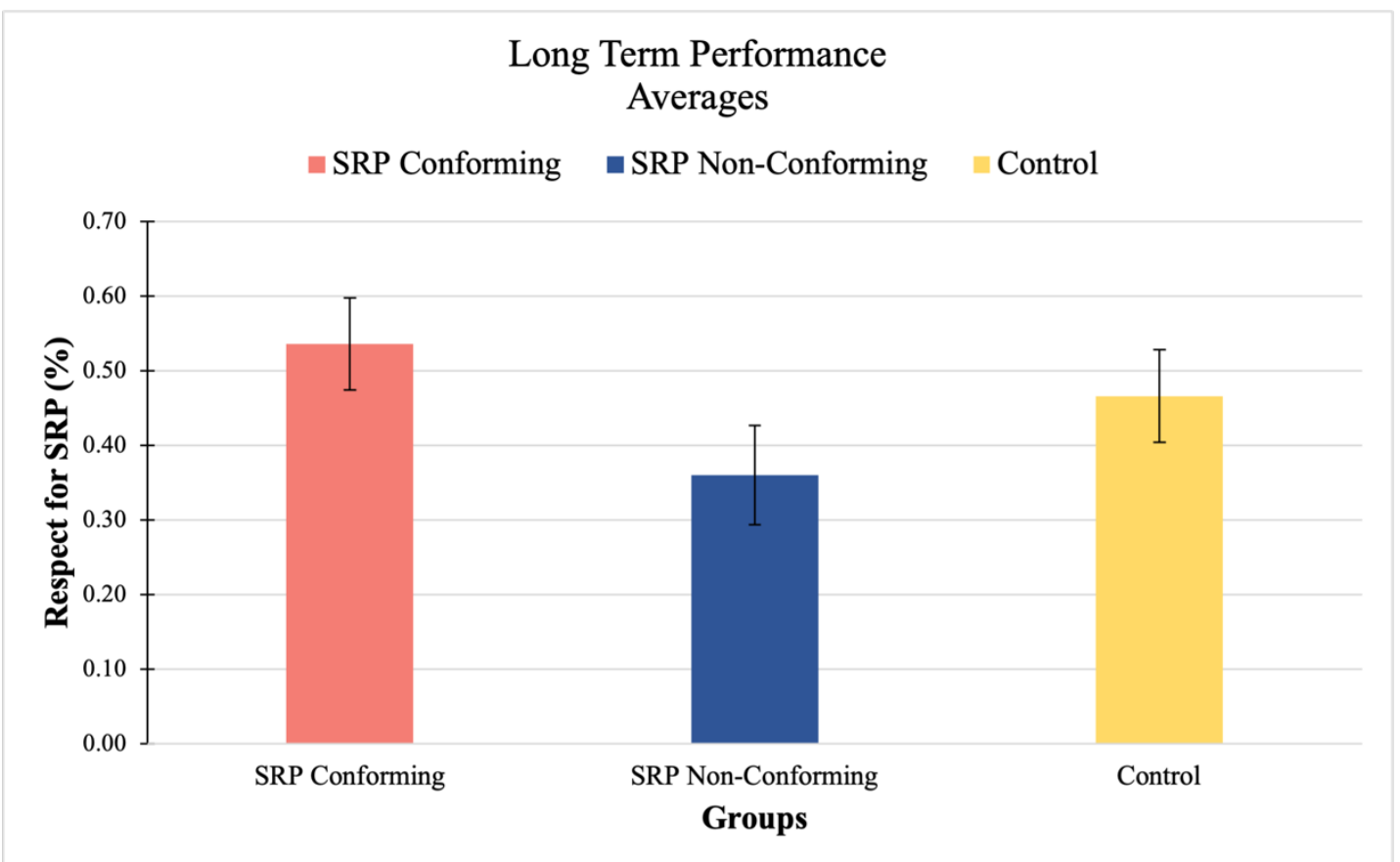

Figure 3. Chart of long-term performance averages for the SRP Conforming, SRP Non-Conforming, and the Control groups.

Nevertheless, these numbers were comparatively high in respect to that same long-term aspect, since for instance, Vinter \& Perruchet (2002) obtained very similar results in their first and only trial, which immediately followed the videos: SRP conforming group (59\%) and SRP non-conforming group (37\%), (See Figure 3). Thus, the implicit learning was still strong following a one-day time period.

Regardless, just like in the short-term performances, the actual effect of the SRP was not very significant, as even the participants who watched the videos in congruity with the principle, only followed so about half the time when asked to execute the task. For the long-term effect, this may have been due to unreliability of memory, since the participants were not shown the videos again and had to recall from what they remembered. In either case, it was to be expected they would follow the principle more so than was recorded. As mentioned before, a possible reason for the low SRP effect, could have been the devices the participants used.

Furthermore, T-Tests were conducted between the SRP conforming, SRP non-conforming and control groups. However, neither of these were significant as they all reported $p>.05$. From this, it can be deduced that the difference among these may have occurred by chance, including factors like the time period after which it followed. 


\subsection{Explicit Awareness}

\section{2a Short Term Awareness}

Lastly, another T-Test was conducted between Group 1 and Group 2, but this time measuring their short-term awareness. To do this, the data from the questionnaire the participants answered was used. The 15 participants in Group 1 $(M=.73, S D=0.2907)$ compared to the 11 of those in Group $2(M=.2954, S D=0.2184)$ also demonstrated a significantly higher compliance with the SRP, $t(24)=4.1935, p<.001$, much like in the short-term performance. This leads to the belief that there is a strong correlation between the implicit performance and explicit awareness.

\subsection{Relationships}

Furthermore, a general positive relationship appeared between implicit performance and explicit awareness, as can be demonstrated by the data. Overall, the participants that attained a higher implicit performance in respect to the SRP, also tended to show a greater percentage of correct awareness regarding the covariation. This was true for both groups that watched the videos. Group 1 (SRP conforming) had a 63\% average of implicit performance in accordance with SRP and 73\% average of explicit awareness in accordance with SRP, whereas Group 2 (SRP non-conforming) had $31 \%$ average of implicit performance in accordance with SRP and 29\% average of explicit awareness in accordance with SRP. Moreover, Vinter and Perruchet also arrived at a similar finding, thus strengthening the claim that there is a relationship between implicit performance and explicit awareness.

To further measure the relationships between both categories: implicit learning through observation and longevity of this effect, an analysis of variance (ANOVA) was conducted (See Table 1). This demonstrated that although there seemed to be a relevantly significant effect of actual implicit learning, $F(1,50)=3.08, \mathrm{p}<0.001$, there appeared to be no pertinent effect of time, $p<0.05$, while there was a marginally significant interaction between the two, $F(2,50)$ $=1.46, \mathrm{p}>0.05$, indicating that it is probable the implicit learning through observation effect becomes weaker with time.

Overall, these results from the Test phase data demonstrate that participants modified their behavior of a motor task, after having watched videos of the inverted covariation of a drawing task to a relevant extent without explicit knowledge. This effect was mostly prominent directly following the observations, as within a short time interval (1 day), it began to diminish. Similar results were reported by Burke and Roodenrys (2000), who also demonstrated a change in behavior without explicit knowledge in a SRT task. However, it is important to note that there can be significant differences in association between implicit performance and explicit awareness among individual subjects, as was shown by Willingham et al. (1989). These implications, along with other limitations and the importance of the study will be discussed further in depth next.

Table 1. ANOVA between SRP and Time

\begin{tabular}{cccccc}
\hline Source & $S S$ & $d f$ & $M S$ & $F$ & $p$-value \\
\hline SRP & 0.7806 & 1 & 0.7806 & 16.13 & 0.0002 \\
Time & 0.0066 & 1 & 0.0066 & 0.14 & 0.7123 \\
Interaction & 0.0642 & 1 & 0.0642 & 1.33 & $\mathbf{0 . 2 5 5 3}$ \\
Error & 2.3231 & 48 & 0.0484 & & \\
Total & 3.1828 & 51 & & & \\
\hline
\end{tabular}




\section{Discussion}

As was evident in the results, there was a modification in the participants' performance after having watched videos that contained variations of a drawing principle. This was corroborated by both the implicit performance and explicit knowledge task data. Overall, this finding indicates that implicit learning of a motor behavior through observation took place in the middle-school students. This relates to similar conclusions of other studies, including Vinter \& Perruchet (2002). Furthermore, this judgement can be discussed in the wider literature of the behavioral field. There had been a gap in the understanding of whether implicit learning can take place through observation alone, that is without a motor component, or explicit influences. Since the prime study upon which the presented method was built had participants use eye movements to an extent, this study omitted that motor movement and provided an alternative.

There were some studies that claimed learning by observation was mostly due to explicit influences (Howard, et al., 1992; Kelly, et al., 2003). The present study challenges that view, since its entire premise was based on using a method that exempted explicit influences, like the neutral parameter procedure. Therefore, the current findings suggest that implicit learning can take place without the use of conscious knowledge and do so through observation alone. Other studies do agree with this conclusion (Nissen \& Bullemer, 1987; Reber, 1993). Bird, et al. (2005) found that no overt action was necessary during observation in order for implicit learning to take place. This further connects with the aforementioned neuropsychological principle known as early mediation and although the present study cannot speak on the role of the mirror neuron system, its role is highly plausible.

\subsection{Limitations and Implications}

It is important to address some potential limitations that arose in the current study, and that should be taken into consideration for both generalizing conclusions and future research. For example, as was mentioned earlier in the Methods, in order to maximize hygiene, the participants each used their own device. Therefore, there were all types of pointers, including touchscreen, trackpad and mouse. Although there has been no research regarding the particular drawing task at hand and the effect of method of control, it is suspected this could have impacted the results since perhaps depending on the device the participants were using, they may have felt more inclined to draw a certain way.

Thus, further research should consider using a standard device to account for this factor. Furthermore, if feasible, the current method could be recreated while also taking into account the MNS and in addition to the neutral parameter procedure, using neuroimaging techniques.

More interestingly, the effect of time merits further study. Although at first the T-Test seemed to indicate the effect diminished over time, the ANOVA later showed it did not. However, if done on a larger population size, this marginal significance may differ. Thus, the effect of time on a bigger population size might be considered. Lastly, longer than one day intervals should be used in future research to more accurately measure longevity of the effect.

The potential applications of this knowledge are vast. Given that we are constantly developing and growing, implicit learning plays a big role in understanding modeling in general. In particular, since the present study focused on middle-school students, with the expanding research in this field, the new opportunities that arise in and outside the classroom are exciting.

\section{Conclusion}

Ultimately, this study was able to accomplish its primary goal of analyzing the extent to which implicit learning can take place through observation alone in middle school students, in both the short and long term. It was found that there was, in fact, a significant indication that a change in behavior had occurred to those students that were exposed to variations of the SRP. Thus, implicit learning was demonstrated to a significant extent in both the short- and long- 
term. This supports the hypothesis that participants would show evidence of implicit learning through observation alone, even without a motor component.

\section{Acknowledgments}

I would like to thank those who I worked with during the course of this study. My AP Capstone Research teacher, Mrs. Janette Sierra, who guided me through the investigation process until the very end, even when at times during my first steps I struggled to see the final result. For this I am grateful. To cognitive neuroscientist and mentor Maryam Vaziri Pashkam, who despite all their expertise was more than willing to share their knowledge with me. Thank you for answering all my questions and providing professional feedback. To Mrs. Michelle Roche, my AP Psychology teacher, whose passion in the subject inspired me, thank you for the honest advice. Thank you to all the PPMH school staff who allowed for me to conduct my experiment, to the participants for their collaboration, and my peers for their comfort. Thank you to all the educators who paved the way and encouraged me to pursue my goals, as difficult as they seem to be. Finally, I would like to thank my family for all their moral support, now and always.

\section{References}

Bandura, A. (1971) Social Learning Theory. General Learning Press.

Bandura, A. (1986) Social foundations of thought and action: A social cognitive theory. Prentice-Hall.

Bird, G., Osman, M., Saggerson, A., \& Heyes, C. (2005). Sequence learning by action, observation and action observation. British Journal of Psychology, 96(Pt 3), 371-388.

Blakemore, C. (1977). Mechanics of the Mind. Cambridge University Press.

Burke, D., \& Roodenrys, S. (2000). Implicit learning in a simple cued reaction-time task. Learning and Motivation, 31. 364-380.

Caspers, S., Geyer S., Schleiher, A., Mohlberg, H., Amunts, K., \& Zilles, K., (2006). The human inferior parietal cortex: cytoarchitectonic parcellation and interindividual variability. Neuroimage 33.2 , 430 448 .

di Pellegrino, G., Fadiga, L., Fogassi, L., Gallese, V., \& Rizzolatti, G., (1992). Understanding motor events: a neurophysiological study. Experimental Brain Research 91, 176-180.

Heyes, C. M., \& Foster, C. L. (2002). Motor learning by observation: Evidence from a serial reaction time task. The Quarterly Journal of Experimental Psychology Section A, 55(2), 593-607.

Howard, J. H., Jr, Mutter, S. A., \& Howard, D. V. (1992). Serial pattern learning by event observation. Journal of Experimental Psychology. 18(5), 1029-1039.

Kanakogi, Y., \& Itakura, S. (2010). The link between perception and action in early infancy: from the viewpoint of the direct-matching hypothesis. Journal of Psychology Research 52, 121-131. 
Kelly, S., Burton, A., Riedel, B., \& Lynch, E. (2003). Sequence learning by action and observation: Evidence for separate mechanisms. British Journal of Psychology 94. 355-372.

Lodish, H., Berk A., Zipursky, L., Matsudaira, P., Baltimore, D., \& Darnell, J. (2000). Molecular Cell Biology. 4th edition. W. H. Freeman.

Maslovat, D., Hodges, N.J., Krigolson, O.E., Handy, T. (2010). Observational practice benefits are limited to perceptual improvements in the acquisition of a novel coordination skill. Experimental Brain Research 204, 119-130.

Nissen, M. J., \& Bullemer, P. (1987). Attentional requirements of learning: Evidence from performance measures. Cognitive Psychology, 19(1), 1-32.

Reber, A. S. (1993). Implicit Learning and Tacit Knowledge: An Essay on the Cognitive Unconscious. Oxford University Press.

Rizzolatti, G., Fogassi L., \& Gallese, V. (2001). Neurophysiological mechanisms underlying the understanding and imitation of action. National Reviews Neuroscience; 2(9):661-670.

Rizzolatti, G., Cattaneo, L., Fabbri-Destro, M., \& Rozzi, S. (2014). Cortical mechanisms underlying the organization of goal-directed actions and mirror neuron-based action understanding. Physiological Reviews, 94(2), 655-706.

Rüsseler, J., \& Rösler, F. (2000). Implicit and explicit learning of event sequences: evidence for distinct coding of perceptual and motor representations. Acta Psychologica, 104(1), 45-67.

Spielman, M., Dumper, K., Jenkins, W., Lovett, M., \& Perlmutter, M. (2014). Psychology. OpenStax.

van Sommers, P. (1984). Drawing and Cognition: Descriptive and Experimental Studies of Graphic Production Processes. Cambridge University Press.

Vinter, A., \& Perruchet, P. (1999). Isolating unconscious influences: The neutral parameter procedure. The Quarterly Journal of Experimental Psychology Section A, 52(4), 857-875.

Vinter, A., \& Perruchet, P. (2002). Implicit motor learning through observational training in adults and children. Memory \& Cognition 30, 256-261.

Vogt, S. (1995). On relations between perceiving, imagining and performing in the learning of cyclical movement sequences. British Journal of Psychology, 86(2), 191-216.

Vogt, S., \& Thomaschke, R. (2007). From visuo-motor interactions to imitation learning: behavioural and brain imaging studies. Journal of Sports Sciences, 25(5), 497-517.

Willingham, D. B., Nissen, M. J., \& Bullemer, P. (1989). On the development of procedural knowledge. Journal of Experimental Psychology: Learning, Memory, \& Cognition, 15, 1047-1060.

Vaziri-Pashhkam, M. (2021) Personal communication [Email and video conferences]. 